Pacific Journal of Mathematic 


\section{ON THE STRUCTURE OF HYPER-REAL $z$-ULTRAFILTERS}

\section{J. Glenn Brookshear}

This paper investigates the structure of hyper-real $z$ ultrafilters on completely regular, Hausdorff spaces in an attempt to describe their structure in manageable terms. A consequence of this investigation is a scheme for classifying these $z$-filters based on the complexity of their structure. It is shown that the real numbers with the usual topology exhibit hyper-real $z$-ultrafilters within each category of the classification. The paper closes with a discussion of how the action of $z$-filters in one category influence those in the other categories with particular applications to the study of $C^{\sharp}(X)$.

The study of $\beta X$, the Stone-Čech compactification of the completely regular Hausdorff space $X$, has proven to be a useful yet complex subject. Many results in the area (for example, in the nonhomogeneity question of $\beta X \backslash X)$ have had their foundations in an understanding of the structure of the $z$-ultrafilters on $X$. For example, remote points, although defined as points in $\beta X \mid \nu X$ which are not in the $\beta X$-closure of a discrete subspace of $X$, were first shown to exist by showing the existence of a free $z$-ultrafilter no element of which is nowhere dense.

This paper investigates the structure of hyper-real $z$-ultrafilters on completely regular, Hausdorff spaces. The object is to describe these filters (or, more accurately, their bases) in terms that provide an insight into their structure. This is done by observing that every hyper-real $z$-ultrafilter has the structure of a free ultrafilter on $\boldsymbol{N}$, the discrete space of natural numbers, underlying it. This underlying structure is formalized in terms of a skeleton. It is shown that many hyper-real $z$-ultrafilters can be realized as being built up from a skeleton using other $z$-ultrafilters (one for each element in $\boldsymbol{N}$ ) in a way which closely resembles that of a subdirect product. In such cases, the original $z$-ultrafilter is said to be real-decomposable or hyper-decomposable depending on whether or not these other $z$-ultrafilters can be chosen to be real $z$-nltrafilters. Although there are hyper-real $z$-ultrafilters that defy such decomposition, the present theory provides insight into the structure of a significant set of hyper-real $z$-ultrafilters as shown by the fact that the set of points associated with the decomposable $z$-ultrafilters is dense in $\beta X \mid \nu X$. A consequence of the present theory is a classification of the hyper-real $z$-ultrafilters into three classes; namely, real-decomposable, hyper- 
decomposable, and nondecomposable.

Ample background can be obtained from [3] and [7]. All spaces are assumed to be completely regular, Hausdorff. The $z$-ultrafilter on $X$ associated with a point $p \in \beta X$ will be denoted by $A^{p}$. Such a distinction between the points of $\beta X$ and the $z$-ultrafilters on $X$, although theoretically unnecessary, is maintained since the emphasis here is on the structure of the filters.

DEFINITION 1. A skelton on a space $X$ is a free ultrafilter $\mathscr{N}$ on $N$ together with a countable collection $\left\{W_{i}: i \in N\right\}$ of mutually disjoint zero-sets such that the function that has value $i$ at each point of $W_{i}$ can be extended continuously to $X$. Such a skelton will be denoted by $\left(\mathscr{N},\left\{W_{i}: i \in N\right\}\right)$. It is said to be a skelton of the hyper-real $z$-ultrafilter $\mathscr{Z}$ provided $N \in \mathscr{N}$ if and only if $\bigcup_{i \in N} W_{i} \in \mathscr{L}$, i.e., the $z$-filter generated by $\left\{\bigcup_{i \in N} W_{i}: N \in \mathscr{N}\right\}$ is in $\mathscr{z}$.

One will recognize that saying $\left(\mathscr{N},\left\{W_{i}: i \in N\right\}\right)$ is a skelton for $A^{p}$ is equivalent to saying $p \in \operatorname{cl}_{\beta X}\left(\bigcup_{i \in N} W_{i}\right)$ if and only if $N \in \mathscr{N}$, i.e., $\mathscr{N}=\left\{N: N \leqq N\right.$ and $\left.p \in \operatorname{cl}_{\beta X}\left(\bigcup_{i \in N} W_{i}\right)\right\}$.

Observe that if the points of a $C$-embedded copy of $N$ are themselves zero-sets then, by letting each $W_{i}$ be one of these points, one can obtain $2^{c}$ skeltons from the copy of $N$ (one from each free ultrafilter on $\boldsymbol{N}$ ). On the other hand, if each $W_{i}$ in a single point (identified as $p_{i}$ ), then these points form a $C$-embedded copy of $\boldsymbol{N}$. (If $\left\{r_{1}: i \in N\right\}$ is a countable subset of $R$, the space of real numbers with the usual topology, there must be a $g \in C(X)$ with $g\left(p_{i}\right)=r_{i}$ for each $i \in N$. This is obtained by choosing $h \in C(R)$ so that $h(i)=r_{i}$ and, by the properties of a skelton, picking $f \in C(X)$ with $f\left(p_{i}\right)=i$ for each $i \in N$. Now define $g=h \circ f$.)

Next it is shown that every hyper-real $z$-ultrafilter has a skelton. The idea here is quite similar to that of 2.1 in [2].

THEOREM 2. Every hyper-real z-ultrafilter has a skelton.

Proof. Let $\mathscr{z}$ be a hyper-real $z$-ultrafilter. Then there is a nonnegative $f \in C(X)$ which is unbounded on each $Z \in \check{z}$. Observe that if $A_{i}$ an $B_{i}$ are the closed intervals [2i-2,2i-1] and [2i-1,2i] respectively for each $i \in N$, then at least one of the zero-sets $\bigcup_{i \in N} f^{\leftarrow}\left[A_{i}\right]$ or $\bigcup_{i \in N} f^{\leftarrow}\left[B_{i}\right]$ must belong to $\not$. Let $W_{i}=f^{\leftarrow}\left[A_{i}\right]$ or $f^{\leftarrow}\left[B_{i}\right]$ respectively for each $i \in N$. Then $\left\{W_{i}: i \in N\right\}$ is a countable collection of mutually disjoint zero-sets. Moreover, there is a $g \in C(X)$ such that $g(x)=i$ for each $x \in W_{i}$.

Now for each $Z \in \mathscr{Z}$ let $N_{Z}$ be the subset of natural numbers $i$ for which $Z \cap W_{i} \neq \varnothing$ (which must occur for an infinite subset 
of $N$ since $\bigcup_{i \in N} W_{i} \in \mathscr{Z}$ and $f$ is bounded on each $W_{i}$ ). Then $\mathscr{N}=\left\{N_{Z}: Z \in \mathscr{Z}\right\}$ is a free ultrafilter on $N$, and $N \in \mathscr{N}$ if and only if $\bigcup_{i \in N} W_{i} \in \mathscr{Z}$. Consequently, $\left(\mathscr{N},\left\{W_{i}: i \in N\right\}\right)$ is a skeleton for $\mathscr{x}$.

Theorem 2 has several consequences that will not be pursued here. One is that no hyper-real $z$-ultrafilter can have a base of connected zero-sets. In fact, any base must contain zero-sets with an infinite number of components. Another is the fact that for any hyper-real $z$-ultrafilter there is a function (one which is $i$ on each $\left.W_{i}\right)$ whose range on each member of some base of the filter has cardinality equal to that of $N$. (Each member of a base must meet infinitely many $W_{i}$ 's.)

The consequence of Theorem 2 that is relevant here is the fact that any hyper-real $z$-ultrafilter has as its fundation the structure of a free ultrafilter on $N$. It follows that the simplest hyper-real $z$-ultrafilters are those with no additional structure, e.g., the free ultrafilters on $N$ itself.

To motivate the following theorem consider a hyper-real $z$-ultrafilter on $\boldsymbol{R}$ with the skelton $\left(\mathscr{N},\left\{\left\{r_{i}\right\}: i \in N\right\}\right)$. Then the collection of zero-sets of the form $\bigcup_{i \in N}\left(Z_{i} \cap\left\{r_{i}\right\}\right)\left(=\bigcup_{i \in N}\left\{r_{i}\right\}\right.$ ), where $N \in \mathscr{N}$ and each $Z_{i}$ is a zero-set in the real $z$-ultrafilter $A^{r_{i}}$, is a base for $\mathscr{Z}$. It follows that the structure of $\mathscr{Z}$ (especially a base for $\mathscr{\%}$ ) is no more complex than the structure of $\mathscr{N}$.

TheOREm 3. Let $\left(\mathscr{N},\left\{W_{i}: i \in N\right\}\right)$ be a skeleton on $X$ and for each $i \in N$ let $\mathscr{y}_{2}$ be a z-ultrafilter on $X$ containing $W_{i}$. Then $\mathscr{F}_{0}=$ $\left\{\bigcup_{i \in N}\left(Z_{i} \cap W_{i}\right): Z_{i} \in \mathscr{Z}_{i}\right.$ and $\left.N \in \mathscr{N}\right\}$ is a base for a hyper-real $z$ ultrafilter $\mathscr{Z}$ on $X$ with skeleton $\left(\mathscr{N},\left\{W_{i}: i \in N\right\}\right)$.

Proof. Since the sets $W_{i}$ are the zero-sets in a skeleton, each member of $\mathscr{Z}_{0}$ is a zero-set. Furthermore, $\mathscr{Z}_{0}$ is a base for a $z$-filter since if $\bigcup_{i \in N_{1}}\left(Z_{i, 1} \cap W_{i}\right)$ and $\bigcup_{i \in N_{2}}\left(Z_{i, 2} \cap W_{i}\right)$ are in $\mathscr{L}_{0}$ then $\bigcup_{i \in N_{i} \cap N_{2}}\left(Z_{i, 1} \cap Z_{i, 2} \cap W_{i}\right) \in \mathscr{Z}_{0}$ is contained in their intersection.

Suppose now that $Z$ is a zero-set that meets every member of $\mathscr{Z}_{0}$. Then for some $N \in \mathscr{N}, Z \in \mathscr{Z}_{i}$ for each $i \in N$. Thus, $\bigcup_{i \in N}\left(Z \cap W_{i}\right)$ is in $\mathscr{Z}_{0}$ and is contained in $Z$. Consequently, $\mathscr{Z}$ is a $z$-ultrafilter. Finally, $\mathscr{Z}$ is hyper-real since there is a function that is $i$ on each $W_{i}$ and thus unbounded on every member of $\mathscr{Z}$.

The fact that $\left(\mathscr{N},\left\{W_{i}: i \in N\right\}\right)$ is a skelton for follows directly from the definition of $\because$.

Definition 4. A hyper-real $z$-ultrafilter $\&$ that can be defined in terms of a skeleton as in Theorem 3 will be called decomposable. 
If for some skelton of $\mathscr{F}$ each $\mathscr{\varkappa}_{i}$ can be chosen to be a real $z$ ultrafilter then $\mathscr{Z}$ will be called real-composable. If $\mathscr{Z}$ is decomposable but not real-decomposable, it will be called hyperdecomposable.

Thus, a hyper-real $z$-ultrafilter is decomposable if it has a base whose trace on each $W_{i}$ in one of its skeltons is a $z$-filter base (disregarding the occurrences of $\varnothing$ ). It is real-decomposable if each of these filter bases is fixed. Observe that in Theorem $3 \not \mathscr{F} \neq \mathscr{K}_{i}$ for each $i \in \boldsymbol{N}$. Thus, a hyper-real $z$-ultrafilter will never occur in its own decomposition.

Since the real $z$-ultrafilters are the simplest $z$-ultrafilters (each being the collection of zero-sets containing a given $p \in U X)$, the realdecomposable $z$-ultrafilters are the least complex hyper-real $z$-ultrafilters. In particular, every hyper-real $z$-ultrafilter on $N$ is realdecomposable. More generally, if $X$ is realcompact, then the structure of a real-decomposable $z$-ultrafilter is very closely related to a free ultrafilter on the set of points associated with the $z$ ultrafilters into which it is decomposed. Such a relationship is most explicit when these points themselves are zero-sets as already observed in the case of $\boldsymbol{R}$. The following characterization of decomposable $z$-ultrafilters extends this line of thought.

TheOREm 5. A point $p \in \beta X \backslash \cup X$ corresponds to a decomposable $z$-ultrafilter if and only if $p$ is the $\beta X$-closure of a countably infinite set $D$ that is $C$-embedded in $D \cup \cup X$. It is associated with a realdecomposable z-ultrafilter if and only if $D$ can be chosen in $\cup X$.

Proof. If $A^{p}$ is decomposable into the filter $A^{d_{2}}$ for $i \in N$ then $\left\{d_{i}: i \in N\right\}$ is the desired set $D$. Note that it is $C$-embedded in $D \cup \cup X$ since each $d_{i} \in \operatorname{cl}_{\beta X} W_{i}$ for some skelton (. $\mathscr{T},\left\{W_{i}: i \in N\right\}$ ). Moreover, if $A^{p}$ is real-decomposable $D \subseteq \cup X$.

Conversely, suppose $p \in \operatorname{cl}_{\beta X} D$ where $D=\left\{d_{i}: i \in N\right\}$ is a $C$ embedded copy of $N$ in $D \cup \cup X$. Pick $f \in C(D \cup \cup X)$ such that $f\left(d_{i}\right)=i$ for each $i \in N$ and define $W_{i}=u X \cap f^{\leftarrow}[i-1 / 3, i+1 / 3]$. Now let $\mathscr{N}$ be the ultrafilter on $N$ consisting of the sets $N_{Z}$ where $Z \in A^{p}$ and $N_{Z}=\left\{i: Z \cap W_{i} \neq \varnothing\right\}$. Then $A^{p}$ is decomposable in terms of the skelton $\left(\mathscr{N},\left\{W_{i}: i \in N\right\}\right)$ and the $z$-ultrafilers $A^{d_{i}}$.

Proposition 6. If $\mathscr{2}$ is a real-decomposable and $f \in C(X)$ then $f[Z]$ is countable for some $Z \in \mathscr{Z}$.

Proof. If $\mathscr{Z}$ is decomposable into the real $z$-ultrafilters $\mathscr{K}_{i}$ for $i \in N$ using the skelton $\left(\mathscr{N},\left\{W_{i}: i \in N\right\}\right)$, then for each $i \in N$ there 
is a $Z_{i} \in \mathscr{Z}_{i}$ on which $f$ is constant. Thus, $f\left[\bigcup_{i \in N}\left(Z_{i} \cap W_{i}\right)\right]$ is countable and $\bigcup_{i \in N}\left(Z_{i} \cap W_{i}\right) \in \mathscr{Z}$.

An example of a hyper-decomposable $z$-ultrafilter is now at hand. For each $i \in N$ let $W_{i} \subseteq R$ be the union of the closed intervals of the form $\left[2^{n+i}+2(i-1), 2^{n+i}+2(i-1)+1\right]$ where $n \in N$. Then $\left\{W_{i}: i \in N\right\}$ is a mutually disjoint family of zero-sets in $\boldsymbol{R}$ for which there is an $f \in C(\boldsymbol{R})$ with $f(x)=i$ if $x \in W_{i}$. Moreover, for each $i \in N$ there is a $z$-ultrafilter $\mathscr{z}_{i}$ containing $W_{i}$ and consisting only of sets with infinite measure. (To obtain such a $z$-ultrafilter let $g_{i} \in$ $C(\boldsymbol{R})$ map each interval $\left[2^{n+i}+2(i-1), 2^{n+i}+2(i-1)+1\right]$ of $W_{i}$ linearly onto the interval $[n, n+1]$. Then for each zero-set $Z$ of $\boldsymbol{R}, \boldsymbol{g}_{i}^{\leftarrow}[Z] \cap W_{i}$ is a zero-set. Thus, the existence of $\mathscr{Z}_{i}$ above follows from the existence of a $z$-ultrafilter on $\boldsymbol{R}$ containing only sets of infinite measure [3,6U.5].) Now pick $\mathscr{N}$ to be a free ultrafilter on $\boldsymbol{N}$ and $\not{z}$ be the hyper-real $z$-ultrafilter on $\boldsymbol{R}$ with base $\left\{\bigcup_{i \in N}\left(Z_{i} \cap W_{i}\right): Z_{i} \in \mathscr{Z}_{i}\right.$ and $\left.N \in \mathscr{N}\right\}$. Then $\mathscr{\mathscr { P }}$ is decomposable but each of its members has infinite measure. Thus, the function mapping each $\boldsymbol{r} \in \boldsymbol{R}$ to itself has uncontable range on each zero-set in $\mathscr{L}$ so, by Proposition $6, \mathscr{L}$ is not real-decomposable.

The preceding example also shows that a hyper-real $z$-ultrafilter may be decomposed in terms of one of its skeltons but not in terms of another. Consider the skelton of the above $z$-ultrafilter obtained via the proof of Theorem 2 with the function $f(r)=|r|$. Any hyper-real $z$-ultrafilter decomposable in terms of this skelton must be real-decomposable since each zero-set in the skelton is compact.

Therefore, to demonstrate the existence of a nondecomposable $z$-ultrafilter it is not sufficient to exhibit a hyper-real $z$-ultrafilter that is not decomposable in terms of one of its skeltons. However, the existence of nondecomposable $z$-ultrafilters follows from Theorem 5 , and the existence of remote points that are also $P$-points in $\beta X \mid v X$. Indeed it is shown in [6] that there is a dense set of $2^{C}$ points in $\beta \boldsymbol{R} \backslash \boldsymbol{R}$ that are both remote points of $\beta \boldsymbol{R}$ and $P$-points of $\beta \boldsymbol{R} \backslash \boldsymbol{R}$. It therefore follows from Theorem 5 that the $z$-ultrafilters associated with these points are not decomposable. Observe that both conditions are required since the hyper-decomposable $z$-ultrafilter following Proposition 6 is associated with a remote point [7.4.40(b)] and all the $P$-points in $\beta \boldsymbol{N} \backslash \boldsymbol{N}$ are associated with real-decomposable $z$-ultrafilters.

Thus, there are cases where nondecomposable $z$-ultrafilters are abundant. On the other hand, if $\rho X$ denotes the subspace of $\beta X$ consisting of $U X$ and the points associated with real-decomposable $z$-ultrafilters, then $\rho X$ is pseudocompact. (If a function were unbounded on $\rho X$ its restriction to $\cup X$ would be unbounded on a $C$ - 
embedded copy of $N$. This restriction therefore could not be extended to $\rho X$ by Theorem 5 which is an obvious contraction.) Thus, by 8.3 of [7], $\rho X \mid \cup X$ is dense in $\beta X \mid \cup X$. In other words, real-decomposable $z$-ultrafilters are always abundant when hyper-real $z$-ultrafilters are present.

This denseness of real-decomposable $z$-ultrafilters together with their relationship to the $C$-embedded copies of $N$ in $\cup X$ indicate that their properties should have a strong influence on the properties of the other $z$-ultrafilters and thus on the space in general. The following theorem is an example of this phenomenon. (If $f: X \rightarrow Y$ then the image of a $z$-filter $\mathscr{Z}$ of $X$ will be the collection of zero-sets in $Y$ whose preimages are in $\mathscr{Z}$.)

THeOREM 7. If $f: X \rightarrow Y$ is a continuous surjection and each point of $Y$ is a zero-set, then the following are equivalent.

(i) The image of each real-decomposable z-ultrafilter on $X$ is a fixed z-ultrafilter.

(ii) In each z-ultrafilter on $X$ there is a zero-set on which $f$ is constant.

(iii) The image of each z-ultrafilter on $X$ is a fixed $z$-ultrafilter.

(iv) The image of every zero-set is compact.

Proof. (i) implies (ii). Since each point of $Y$ is a zero-set, (i) implies that each real-decomposable $z$-ultrafilter contains a zero-set on which $f$ is constant. Suppose $f$ is not constant on any zero-set in $A^{p}$ for some $p \in \beta X \backslash \rho X$. Then the image of each zero-set in $A^{p}$ must be infinite. Consequently, if $\left\{W_{i}: i \in N\right\}$ is the collection of zero-sets in a skelton for $A^{p}$, then a point $d_{i}$ can be chosen in each $W_{i}$ so that the image of the set $\left\{d_{i}: i \in N\right\}$ is infinite. Let $D$ be a $C$-embedded copy of $\boldsymbol{N}$ obtained from $\left\{d_{i}: i \in N\right\}$ by discarding any $d_{j}$ such that $f\left(d_{i}\right)=f\left(d_{j}\right)$ for some $i<j$. Then $f$ cannot be constant on any zero-set in a real-decomposable $z$-ultrafilter associated with a point in $\operatorname{cl}_{\beta X} D$.

(ii) implies (iii). If $p \in \beta X$ and $Z \in A^{p}$ with $f[Z]=\{y\}$, then the image of $A^{p}$ is $A^{y}$.

(iii) implies (iv). First extend $f$ to $f^{\beta}: \beta X \rightarrow \beta Y$ and observe, by (iii) that $Y=\beta Y$. Thus, if $Z$ is a zero-set in $X$, then $f^{\beta}\left[\operatorname{cl}_{\beta_{X}} Z\right]$ is a compact subset of $Y$. Moreover, if $p \in \operatorname{cl}_{\beta_{X}} Z \backslash Z$, then both $Z$ and $f \leftarrow\left[f^{\beta}(p)\right]$ must be in $A^{p}$. Consequently, there is an $x \in Z$ such that $f(x)=f^{\beta}(p)$ so $f[Z]=f^{\beta}\left[\operatorname{cl}_{\beta X} Z\right]$.

(iv) implies (i). Extend $f$ to $f^{\beta}: \beta X \rightarrow \beta Y=Y$. Let $p \in \rho X$ and $y=f^{\beta}(p)$. Since $\{y\}$ is a zero-set in $Y, f^{\leftarrow}[\{y\}]$ is a zero-set in $X$. The proof is completed by showing $f^{\leftarrow}[\{y\}] \in A^{p}$ since this implies the image of $A^{p}$ is $A^{y}$. Suppose that $Z \in A^{p}$. Since $f[Z]$ is compact, it is closed and thus $f^{\beta+}[f[Z]]$ is a closed set containing $Z$. But $p \in \operatorname{cl}_{\beta_{X}} Z$ 
so $p \in f^{\beta \leftarrow}[f[Z]]$. Consequently, $y \in f[Z]$ so $Z \cap f^{\leftarrow}[\{y\}] \neq \varnothing$. Therefore, $f^{\leftarrow}[\{y\}] \in A^{p}$.

Consider now the ring $C^{\sharp}(X)$ consisting of those $f \in C(X)$ whose image $M(f)$ in the residue class ring $C(X) / M$ is real for every maximal ideal $M$ in $C(X)$. The basic properties of this ring are discussed in [1] and [5], and its relationship to the Freudenthal compactification is investigated in [4]. The following is a list of conditions appearing in either [1] or [5] that are equivalent to $f \in C^{\sharp}(X)$.

(A) Every $z$-ultrafilter on $X$ has a member on which $f$ is constant.

(B) $f \in C^{*}(X)$ and the image of every $z$-ultrafilter on $X$ is a $z$ ultrafilter. of $N$.

(C) $f \in C^{*}(X)$ and $f[D]$ is finite for every $C$-embedded copy $D$

(D) $f \in C^{*}(X)$ and $f[Z]$ is closed for every zero-set $Z$ in $X$.

One will observe that the equivalence of these conditions is a direct result of Theorems 5 and 7 . Moreover, condition $\mathrm{C}$ shows that the ring $C^{\sharp}(X)$ arises as a natural restriction to the statement of Theorem 6. Finally, Theorem 7 provides the following additional characterization of $C^{\sharp}(X)$.

CoROLlaRY 8. The following are equivalent.

(i) $f \in C^{\sharp}(X)$.

(ii) $M(f)$ is real for each maximal ideal $M$ associated with a real-decomposable z-ultrafilter.

Proof. This is obtained as a direct result of condition (i) and (ii) of Theorem 7 by setting $Y=\boldsymbol{R}$.

\section{REFERENCES}

1. E. Cheo, Note on a subring of $C^{*}(X)$, Canad. Math. Bull., 18 (1975), 177-179.

2. N. J. Fine and L. Gillman, Remote points in $\beta R$, Proc. Amer. Math. Soc., 13 (1962), $29-36$.

3. L. Gillman and M. Jerison, Rings of Continuous Functions, Van Nostland Reinhold Company, New York, 1960.

4. M. Henriksen, An algebraic characterization of the Freudenthal compactification for a class of rimcompact spaces, Proc. Spring Topology Conference-Baton Rouge, 1976, to appear.

5. L. Nel and D. Riordan, Note on a subalgebra of $C(X)$, Canad. Math. Bull., 15 (1972), 607-608.

6. D. Plank, On a Class of Subalgebras of $C(X)$ with Applications to $\beta X / X$, Thesis, University of Rochester, 1966.

7. R. C. Walker, The Stone-Čech Compactification, Springer-Verlag, New York, 1970.

Received March 16, 1979 and in revised form August 7, 1979. 



\section{PACIFIC JOURNAL OF MATHEMATICS}

\section{EDITORS}

DONALD BABBITT (Managing Editor)

University of California

Los Angeles, CA 90024

HUGo RossI

University of Utah

Salt Lake City, UT 84112

C. C. MOORE and ANDREW OGG

University of California

Berkeley, CA 94720
J. DUGUNDJI

Department of Mathematics

University of Southern California

Los Angeles, CA 90007

R. FinN and J. Milgram

Stanford University

Stanford, CA 94305

\section{ASSOCIATE EDITORS}
R. ARENS
E. F. BECKENBACH
B. H. NeumanN
F. WOLF
K. Yoshida

\section{SUPPORTING INSTITUTIONS}

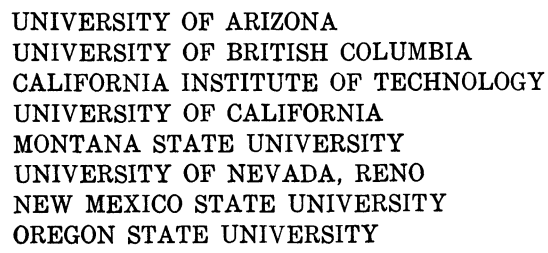

UNIVERSITY OF ARIZONA

UNIVERSITY OF BRITISH COLUMBIA CALIFORNIA INSTITUTE OF TECHNOLOGY

UNIVERSITY OF CALIFORNIA

MONTANA STATE UNIVERSITY

UNIVERSITY OF NEVADA, RENO

NEW MEXICO STATE UNIVERSITY

OREGON STATE UNIVERSITY

\author{
UNIVERSITY OF OREGON \\ UNIVERSITY OF SOUTHERN CALIFORNIA \\ STANFORD UNIVERSITY \\ UNIVERSITY OF HAWAII \\ UNIVERSITY OF TOKYO \\ UNIVERSITY OF UTAH \\ WASHINGTON STATE UNIVERSITY \\ UNIVERSITY OF WASHINGTON
}

The Supporting Institutions listed above contribute to the cost of publication of this Journal, but they are not owners or publishers and have no responsibility for its content or policies.

Mathematical papers intended for publication in the Pacific Journal of Mathematics should be in typed form or offset-reproduced, (not dittoed), double spaced with large margins. Please do not use built up fractions in the text of the manuscript. However, you may use them in the displayed equations. Underline Greek letters in red, German in green, and script in blue. The first paragraph or two must be capable of being used separately as a synopsis of the entire paper. Please propose a heading for the odd numbered pages of less than 35 characters. Manuscripts, in triplicate, may be sent to any one of the editors. Please classify according to the scheme of Math. Reviews, Index to Vol. 39. Supply name and address of author to whom proofs should be sent. All other communications should be addressed to the managing editor, or Elaine Barth, University of California, Los Angeles, California, 90024.

50 reprints to each author are provided free for each article, only if page charges have been substantially paid. Additional copies may be obtained at cost in multiples of 50 .

The Pacific Journal of Mathematics is issued monthly as of January 1966. Regular subscription rate: $\$ 102.00$ a year (6 Vols., 12 issues). Special rate: $\$ 51.00$ a year to individual members of supporting institutions.

Subscriptions, orders for numbers issued in the last three calendar years, and changes of address shoud be sent to Pacific Journal of Mathematics, P.O. Box 969, Carmel Valley, CA 93924, U.S.A Old back numbers obtainable from Kraus Periodicals Co., Route 100, Millwood, NY 10546.

\section{PUBLISHED BY PACIFIC JOURNAL OF MATHEMATICS, A NON-PROFIT CORPORATION}

Printed at Kokusai Bunken Insatsusha (International Academic Printing Co., Ltd.). 8-8, 3-chome, Takadanobaba, Shinjuku-ku, Tokyo 160, Japan. 


\section{Pacific Journal of Mathematics}

Vol. 92, No. $2 \quad$ February, 1981

Bruce Allem Anderson and Philip A. Leonard, Sequencings and Howell designs

Kevin T. Andrews, Representation of compact and weakly compact

operators on the space of Bochner integrable functions . . . . . . . . 257

James Glenn Brookshear, On the structure of hyper-real $z$-ultrafilters . . . . . 269

Frank John Forelli, Jr., A necessary condition on the extreme points of a class of holomorphic functions. II ...................... 277

Richard J. Friedlander, Basil Gordon and Peter Tannenbaum, Partitions of groups and complete mappings ......................... 283

Emden Robert Gansner, Matrix correspondences of plane partitions ......295

David Andrew Gay and William Yslas Vélez, The torsion group of a radical extension ..........................................

André (Piotrowsky) De Korvin and C. E. Roberts, Convergence theorems for some scalar valued integrals when the measure is Nemytskii ...... 329

Takaŝi Kusano and Manabu Naito, Oscillation criteria for general linear ordinary differential equations $\ldots \ldots \ldots \ldots \ldots \ldots \ldots \ldots \ldots \ldots \ldots \ldots \ldots \ldots \ldots \ldots$

Vo Thanh Liem, Homotopy dimension of some orbit spaces .......... 357

Mark Mahowald, $b o$-resolutions . . . . . . . . . . . . . . . . . . . 365

Jan van Mill and Marcel Lodewijk Johanna van de Vel, Subbases, convex

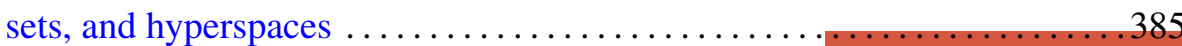

John F. Morrison, Approximations to real algebraic numbers by algebraic numbers of smaller degree $\ldots \ldots \ldots \ldots \ldots \ldots \ldots \ldots \ldots \ldots \ldots \ldots \ldots$

Caroline Series, An application of groupoid cohomology . . . . . . . . . . 415

Peter Frederick Stiller, Monodromy and invariants of elliptic surfaces . . . 433 Akihito Uchiyama, The factorization of $H^{p}$ on the space of homogeneous

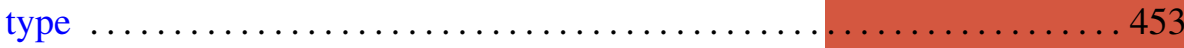

Warren James Wong, Maps on simple algebras preserving zero products.

II. Lie algebras of linear type 